\title{
Limbic encephalitis in a COVID-19 patient?
}

\author{
Luca Chiveri $^{1}$ (1) - Elena Verrengia ${ }^{1}$. Francesco Muscia ${ }^{1}$ - Graziamaria Nuzzaco ${ }^{1}$ - Elisa Raimondi ${ }^{1}$ Emilio Vecchio ${ }^{1}$. \\ Daniela Bompane ${ }^{2} \cdot$ Antonino Mazzone $^{2} \cdot$ Paolo Fociani $^{3} \cdot$ Mario Corbellino $^{4} \cdot$ Alessandro Prelle $^{1}$
}

Received: 23 May 2020 / Revised: 16 August 2020 / Accepted: 4 February 2021 / Published online: 31 March 2021

(c) Journal of NeuroVirology, Inc. 2021

\begin{abstract}
We describe the case of a 79-year-old woman infected by SARS-CoV-2 and purely neurological confusional syndrome without clinically relevant respiratory disease and NMR alterations of the limbic system.
\end{abstract}

Keywords Covid-19 $\cdot$ Limbic encephalitis

A 79-year-old woman came to the emergency room (ER) of our hospital for the acute onset of confusion and amnesia. Her husband referred that the patient had fallen from her bed during the preceding night, but upon direct questioning she did not remember the fact. The patient had no previous neurological history and she was only taking risedronate for osteoporosis. At the time of the first clinical evaluation, she was alert but confused, disoriented and amnesic. No focal neurological signs were evident. Thoracic, cardiac and abdominal physical examinations were normal. She had no fever, respiratory symptoms, muscle pain or fatigue. A complete blood count revealed mild lymphopenia, and arterial blood gas analysis was within the normal range. Radiography of the chest showed bilateral accentuation of the pulmonary interstitium. A nose-pharyngeal swab (NPS) was positive for SARS-CoV-2 RNA, as assessed by reverse transcriptase real-time PCR (rtRT-PCR). The brain CT-scan showed a hypodensity in the left hippocampus. The patient was hospitalized in a COVID-19 dedicated ward area for monitoring. Two days later, she underwent a brain NMR which confirmed an altered signal on the middle and posterior portion of the left hippocampal formation that was

Luca Chiveri

lucachiveri@gmail.com

1 Neurology Departement, ASST Ovest Milanese, Via Papa Giovanni Paolo II 20025, Legnano, Italy

2 Internal Medicine Departement, ASST Ovest Milanese, Via Papa Giovanni Paolo II 20025, Legnano, Italy

3 Pathology Departement, ASST Ovest Milanese, Via Papa Giovanni Paolo II 20025, Legnano, Italy

4 Infectious Departement, ASST FBF-Sacco, Milano, Italy hyperintense in T2 and FLAIR-weighted images, and negative in DWI. A similar but considerably smaller lesion was observed in the posterior right hippocampus (Fig. 1a). The electroencephalogram showed slow waves on the temporal derivations bilaterally. Lumbar puncture was performed on day 4, and showed a cerebrospinal fluid (CSF) with normal protein and glucose concentrations (i.e. protein $36 \mathrm{mg} /$ $\mathrm{dl}$, glucose $59 \mathrm{mg} / \mathrm{dl}$, no cells). The CSF cytopathologic examination revealed a mild nonspecific inflammation with sediment consisting of rare red blood cells and polymorphs, very rare lymphocytes and some macrophages. The search for several bacterial, fungal and viral infectious agents on CSF was negative (Cryptococcus neoformans, Cryptococcus gattii, Enterovirus, Herpes simplex 1 and 2, Human herpesvirus 6, Human parechovirus, Cytomegalovirus, Varicella zoster virus, Adenovirus, Listeria monocytogenes, Neisseria meningitidis, Escherichia coli K1). The CSF was also negative for the presence of neoplastic cells. Furthermore, a panel of antibodies related to autoimmune encephalitides was negative (e.g. anti GAD, AMPA1, AMPA2, CASPR2, NMDAr, GABA B1, GluR3 A/B and DPPX). No SARSCoV-19 RNA was detected by rtRT-PCR in the CSF and the plasma of our patient. During hospitalization, the patient progressively improved with complete regression of confusion after 1 week. However, she remained amnesic for what had occurred at home. During this period of observation, no respiratory sign or symptom developed. A brain NMR was repeated after one week and was normal (Fig. 1b). Considering the rapid improvement she did not receive any treatment and was discharged after 2 weeks.

We present herein the case of a transient, spontaneously reversible confusional syndrome with radiologically 
Fig. 1 a Lesion observed in the posterior right hippocampus. b A brain NMR repeated after 1 week which resulted to be normal
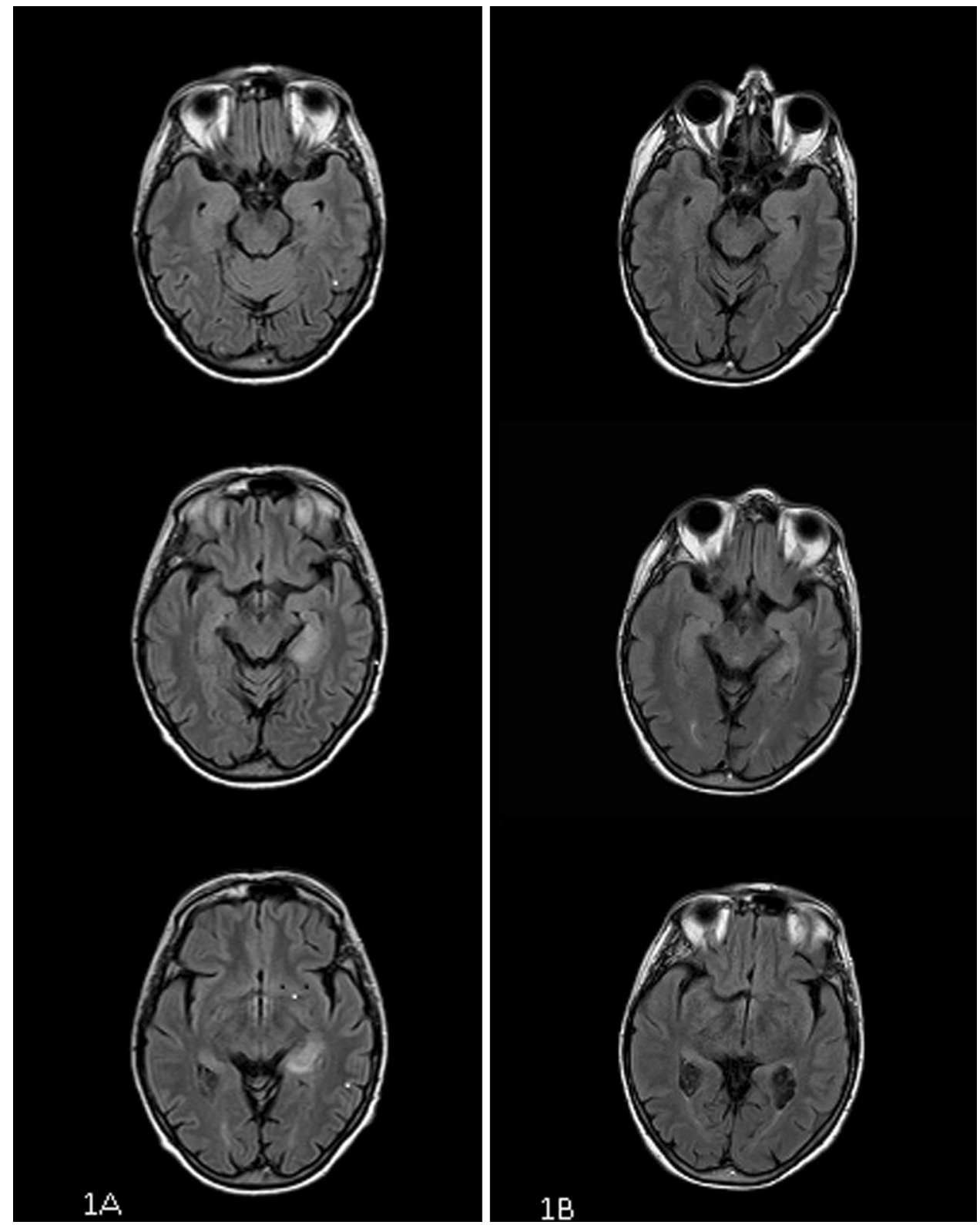

documented hippocampal alterations, reminiscent of limbic encephalitis in a patient with COVID-19 and no clinically relevant respiratory disease.

We tested some antibodies related to autoimmune encephalitides, but all of them were negative; unfortunately, we could not test LGI1 antibody that is often related to limbic encephalitis. On the other hand, the cases of LGI1related limbic encephalitis normally have some differences compared to our case, often having seizures and a longer duration of neuropsychological sequelae Fazekas et al. (2018).

Our patient's clinical manifestations are similar to transient global amnesia, except for confusion and a longer duration of symptoms. Furthermore, in transient global amnesia brain NMR may show small hyperintensity on diffusion-weighted images that are different from those found in our patient Yang et al. (2008). In the former, brain CT scan is usually normal, while in our patient, it showed a hypodense lesion in the left hippocampus. Epileptic manifestations of limbic origin can give rise to NMR alterations in the cerebral cortex similar to those found in our patient, which however are not detectable by CT imaging Cendes et al. (2016). Moreover, an electroencephalogram that was performed during the acute state of confusion did not confirm the epileptic nature of our patient's symptoms.

Our inability to detect SARS-Cov-2 RNA by rtRT-PCR in the CSF cannot be used to disprove a neurotropism of the virus. Further studies, and a heightened attention by the caring physicians to neurological symptoms arising in patients with COVID-19 is needed to address this possibility. 
Right now, we can only speculate about the relationship between virus and the neurological syndrome: can we suppose a direct attack of COVID-19 to temporal lobes or an inflammatory limbic immunological mediated manifestation triggered by the COVID-19 infection? Confusion has been reported in the acute stages of COVID-19. Herein, we documented by NMR alterations of the limbic system in a patient infected by SARS-CoV-2 and purely neurological syndrome, without clinically relevant respiratory disease.

\section{References}

Cendes F et al (2016) Neuroimaging of epilepsy. Handb Clin Neurol 136:985-1014
Fazekas F et al (2018) Systematic Review: Syndromes, Early Diagnosis, and Treatment in Autoimmune Encephalitis. Front Neurol 5 Sept

Yang S et al (2008) Ischemic Evidence of Transient Global Amnesia: Location of the Lesion in the Hippocampus. J Clini Neurolo Jun 4(2):59-66

Publisher's Note Springer Nature remains neutral with regard to jurisdictional claims in published maps and institutional affiliations. 